Bull. Austral. Mațh. Soc.

$32 \mathrm{~F} 05,31 \mathrm{~B} 35$

VoL. 53 (1996) [123-129]

\title{
M-HARMONIC FUNCTIONS WITH M-HARMONIC SQUARE
}

\section{HONG OH KIM}

$\mathcal{M}$-harmonic functions with $\mathcal{M}$-harmonic square are proved to be either holomorphic or antiholomorphic in the unit ball of complex $n$-space under certain additional conditions. For example, if $u$ and $u^{2}$ are $\mathcal{M}$-harmonic in the unit ball of $\mathbb{C}^{2}$ and if $u$ is continuously differentiable up to the boundary then $u$ is either holomorphic or antiholomorphic.

\section{INTRODUCTION}

It is well known and easy to prove that if $u$ and $u^{2}$ are harmonic in an open connected region $\Omega \subset \mathbb{C}$ then at least one of $u$ and $\bar{u}$ is holomorphic in $\Omega$. The analogue of this in the open unit ball $B_{n}$ of $\mathbb{C}^{n}(n>2)$ and with " $\mathcal{M}$-harmonic" in place of harmonic was unexpectedly proved to be false by Ahern and Rudin in [1]. It is not known whether the analogue for $n=2$ is true or not. In this paper we prove the analogue is true under certain additional conditions for $n \geqslant 2$. For example, if $u$ and $u^{2}$ are $\mathcal{M}$-harmonic in the unit ball of $\mathbb{C}^{2}$ and if $u$ is continuously differentiable up to the boundary then $u$ is either holomorphic or antiholomorphic.

We say that a function $u$ is $\mathcal{M}$-harmonic in $B_{n}$ if

$$
\widetilde{\Delta} u(z)=0
$$

for every $z \in B_{n}$, where $\tilde{\Delta}$ is the Moebius-invariant Laplacian:

$$
\widetilde{\Delta} u=\left(1-|z|^{2}\right) \sum_{j, k=1}^{n}\left(\delta_{j k}-z_{j} \bar{z}_{k}\right) \frac{\partial^{2} u}{\partial z_{j} \partial \bar{z}_{k}}
$$

This is related to the ordinary Laplacian $\Delta=\sum \partial^{2} / \partial z_{j} \bar{\partial} z_{j}$ as $(\tilde{\Delta} u)(a)=\Delta(u \circ \phi)(0)$, where $\phi$ is an automorphism of $B_{n}$ mapping the origin to $a$.

It is clear from (1) that all holomorphic or antiholomorphic functions are $\mathcal{M}$ harmonic, as are the pluriharmonic ones. The pluriharmonic functions are those functions that can be represented as a sum of a holomorphic function and an antiholomorphic

Received 23rd March, 1995

Partially supported by KOSEF and TGRC. The author wishes to express his sincere gratitude to Professor P.R. Ahern, discussions with whom made these results possible.

Copyright Clearance Centre, Inc. Serial-fee code: 0004-9729/96 \$A2.00+0.00. 
function. It is an interesting fact that the pluriharmonic functions in the ball $B_{n}$ are those $\mathcal{M}$-harmonic functions that are also ordinary harmonic [3].

We recall that any $\mathcal{M}$-harmonic function $u$ has a spherical harmonic expansion, which converges uniformly on compact subsets of $B_{n}$,

$$
u(z)=\sum_{p, q \geqslant 0} R_{p q}\left(|z|^{2}\right) h_{p q}(z)
$$

where $h_{p q}$ is a homogeneous polynomial of degree $p$ in $z$ and of degree $q$ in $\bar{z}$, and $R_{p q}(t)$ is a hypergeometric function, normalised so that $R_{p, q}(1)=1$ :

$$
R_{p q}(t)=\frac{{ }_{2} F_{1}(p, q, p+q+n ; t)}{{ }_{2} F_{1}(p, q, p+q+n ; 1)} .
$$

See [2]. Finally, we recall the invariant Poisson kernel $P(z, \zeta)$ is given by

$$
P(z, \zeta)=\left(\frac{1-|z|^{2}}{|1-\langle z, \zeta\rangle|^{2}}\right)^{n}, z \in B_{n}, \zeta \in S=\partial B_{n}
$$

[3], and the invariant Poisson integral of a function $u$ on $S$ is given by $P[u](z)$ $=\int_{S} P(z, \zeta) u(z) d \sigma(\zeta)$, where $d \sigma$ is the normalised Lebesgue measure on $S$ with $d \sigma(S)=1$.

\section{THE CASE $n=2$}

It is known in [1] that if $u \in C^{2}\left(\bar{B}_{2}\right)$ and $\bar{\Delta} u=\widetilde{\Delta} u^{2}=0$ then one of $u$ and $\bar{u}$ is holomorphic in $B_{2}$. The smoothness condition $u \in C^{2}\left(\bar{B}_{2}\right)$ is relaxed to $u \in C^{1}\left(\bar{B}_{2}\right)$ in the following main theorem of this paper.

Theorem 1. Suppose $\widetilde{\Delta} u=\widetilde{\Delta} u^{2}=0$ in $B_{2}$. If $u$ is continuously differentiable up to the boundary of $B_{2}$, then one of $u$ and $\bar{u}$ is holomorphic in $B_{2}$.

Proof: Let $T=\bar{z}_{2} \partial / \partial z_{1}-\bar{z}_{1} \partial / \partial z_{2}$ be a tangential Cauchy-Riemann operator and $R=z_{1} \partial / \partial z_{1}+z_{2} \partial / \partial z_{2}$ a radial differential operator on $B_{2}$. Then the hypothesis $\widetilde{\Delta} u=\widetilde{\Delta} u^{2}=0$ implies that

$$
\begin{aligned}
T \bar{T} u & =\left(r^{2}-1\right) R \bar{R} u-\bar{R} u \\
T u \bar{T} u & =\left(r^{2}-1\right) R u \bar{R} u .
\end{aligned}
$$

Since $u \in C^{1}\left(\bar{B}_{2}\right),(4)$ implies that $T u \bar{T} u=0$ on $S$. There are two open subsets $V$ and $W$ of $S$ such that

$$
\begin{aligned}
& \bar{T} u=0 \text { on } V, \\
& T u=0 \text { on } W, \\
& \overline{V \cup W}=S .
\end{aligned}
$$


We fix $\zeta_{0} \in V$ and take $\phi \in C_{c}^{\infty}(V)$ such that $\phi \equiv 1$ near $\zeta_{0}$. We set $u_{1}=\phi u$ and let $U_{1}=P\left[u_{1}\right]$ be the invariant Poisson integral of $u_{1}$. Then

$$
\begin{aligned}
u(z) & =\int_{S} P(z, \zeta) u(\zeta) d \sigma(\zeta) \\
& =\int_{S} P(z, \zeta) \phi(\zeta) u(\zeta) d \sigma(\zeta)+\int_{S} P(z, \zeta)(1-\phi(\zeta)) u(\zeta) d \sigma(\zeta) \\
& =U_{1}(z)+U_{2}(z)
\end{aligned}
$$

We can easily check that

$$
\begin{aligned}
T \bar{T} U_{2}(z) & \rightarrow 0 \\
\bar{R} U_{2}(z) & \rightarrow 0
\end{aligned}
$$

as $z \rightarrow \zeta_{0}$. If we note that

$$
\begin{aligned}
\bar{T} u_{1} & =(\bar{T} \phi) u+\phi \bar{T} u \\
& =\bar{T} \phi u
\end{aligned}
$$

on $S$, we see that $\bar{T} u_{1} \in C^{1}(S)$ and so $T \bar{T} u_{1} \in C(S)$. Since $\widetilde{\Delta} T \bar{T} U_{1}=T \bar{T} \widetilde{\Delta} U_{1}=0$, we also have

$$
T \bar{T} U_{1}(z)=P\left[T \bar{T} u_{1}\right](z) \rightarrow T \bar{T} u_{1}(\zeta), \quad z=r \zeta
$$

as $r \rightarrow 1$. We write $U_{1}(z)=\sum R_{p q}\left(|z|^{2}\right) h_{p q}(z)$ as in (2). An easy computation gives

$$
T \bar{T} U_{1}(z)=-\sum R_{p q}\left(|z|^{2}\right) q(p+1) h_{p q}(z)
$$

Therefore, we have

$$
\int\left|T \bar{T} U_{1}(r \zeta)\right|^{2} d \sigma(\zeta)=\sum_{p, q \geqslant 0} R_{p q}\left(|z|^{2}\right)^{2} q^{2}(p+1)^{2} r^{2 p+2 q} \int\left|h_{p q}(\zeta)\right|^{2} d \sigma(\zeta)
$$

If we let $r \rightarrow 1^{-}$in (10), we get, by (8),

$$
\int\left|T \bar{T} u_{1}(\zeta)\right|^{2} d \sigma(\zeta)=\sum_{p, q \geqslant 0} q^{2}(p+1)^{2} \int\left|h_{p q}(\zeta)\right|^{2} d \sigma(\zeta)<\infty
$$

On the other hand, we have

$$
\bar{R} U_{1}(z)=\sum_{p, q \geqslant 0}\left\{R_{p q}^{\prime}\left(|z|^{2}\right)|z|^{2}+q R_{p q}\left(|z|^{2}\right)\right\} h_{p q}(z)
$$


and so

$$
\begin{aligned}
\int_{S}\left|\bar{R} U_{1}(r \zeta)\right|^{2} d \sigma(\zeta) & =\sum_{p, q \geqslant 0}\left\{R_{p q}^{\prime}\left(r^{2}\right) r^{2}+q R_{p q}\left(r^{2}\right)\right\}^{2} r^{2 p+2 q} \int_{S}\left|h_{p q}(\zeta)\right|^{2} d \sigma(\zeta) \\
& \leqslant \sum_{p, q \leqslant 0} q^{2}(p+1)^{2} \int\left|h_{p q}(\zeta)\right|^{2} d \sigma(\zeta) \\
& <\infty
\end{aligned}
$$

The last inequality comes from (11). Therefore,

$$
\begin{aligned}
& \int_{S}\left|\bar{R} U_{1}(r \zeta)-T \bar{T} u(\zeta)\right|^{2} d \sigma(\zeta) \\
& =\sum_{p, q \geqslant 0}\left\{R_{p q}^{\prime}\left(r^{2}\right) r^{2}+q R_{p q}\left(r^{2}\right) r^{2 p+2 q}-q(p+1)\right\}^{2} \int_{S}\left|h_{p q}(\zeta)\right|^{2} d \sigma(\zeta) \\
& \rightarrow 0
\end{aligned}
$$

as $r \rightarrow 1^{-}$. We can choose a sequence $r_{j} \nearrow 1$ so that $\bar{R} U_{1}(r \zeta) \rightarrow T \bar{T} u_{1}(\zeta)[\sigma]$ almost everywhere on $S$. We can easily see that $\bar{R} U_{1}(r \zeta) \rightarrow \bar{R} u_{1}(\zeta)$ near $\zeta_{0}$ and so we have $T \bar{T} u_{1}(\zeta)=\bar{R} u(\zeta)$ near $\zeta_{0}$ by continuity. We have proved that $\bar{R} u=0$ on $V$ and so on $\bar{V}$ by continuity. Similarly, we can show $R u=0$ on $\bar{W}$. Since $R u \bar{R} u=0$ on $S$, we have, by orthogonality of the $u_{p, q}$ 's,

$$
\begin{aligned}
0 & =\int_{S} \bar{R} u(\zeta) \overline{R u}(\zeta) d \sigma(\zeta) \\
& =\sum p q(p+1)(q+1) \int\left|u_{p q}(\zeta)\right|^{2} d \sigma(\zeta)
\end{aligned}
$$

where $u=\sum u_{p q}$ is the homogeneous expansion of $u$ on $S$ in $L^{2}(\sigma)$. Therefore, $p q=0$ unless $u_{p q} \equiv 0$. This means that $u$ is pluriharmonic in $B_{2}$. If we write $u=f+\bar{g}$ where $f, g$ are holomorphic in $B_{2}$, then

$$
\bar{T} T u=\bar{T} T f=-\left(z_{2} \frac{\partial f}{\partial z_{2}}+z_{1} \frac{\partial f}{\partial z_{1}}\right)
$$

is holomorphic in $B_{2}$ and vanishes on $W$. Suppose $W \neq \phi$. Then $\bar{T} T u \equiv 0$ on $S$ and so $R u \equiv 0$ on $B_{2}$. Therefore $u$ is antiholomorphic in $B_{2}$ by a Theorem of Forelli [3]. Similarly, we can show that $u$ is holomorphic in $B_{2}$ if $V \neq \phi$. This completes the proof.

TheOREM 2. Suppose $\bar{\Delta} u=\tilde{\Delta} u^{2}=0$ in $B_{2}$. If $u$ is holomorphic in one of two variables then one of $u$ and $\bar{u}$ is holomorphic in $B_{2}$. 
ProOF: Suppose $u\left(z_{1}, z_{2}\right)$ is holomorphic in $z_{2}$ in $B_{2} . \tilde{\Delta} u=\widetilde{\Delta} u^{2}=0$ implies that

$$
\begin{aligned}
& \frac{\partial u^{2}}{\partial z_{1} \partial z_{2}}=\left|z_{1}\right|^{2} \frac{\partial^{2} u}{\partial z_{1} \partial \bar{z}_{1}}+\bar{z}_{1} z_{2} \frac{\partial^{2} u}{\partial \bar{z}_{1} \partial z_{2}} \\
& \frac{\partial u}{\partial \bar{z}_{1}}\left(\frac{\partial u}{\partial z_{1}}-\left|z_{1}\right|^{2} \frac{\partial u}{\partial z_{1}}-\bar{z}_{1} z_{2} \frac{\partial u}{\partial z_{2}}\right)=0
\end{aligned}
$$

We have either $\partial u / \partial \bar{z}_{1} \equiv 0$ or

$$
\frac{\partial u}{\partial z_{1}} \equiv\left|z_{1}\right|^{2} \frac{\partial u}{\partial z_{1}}+\bar{z}_{1} z_{2} \frac{\partial u}{\partial z_{2}} .
$$

If $\partial u / \partial \bar{z}_{1} \equiv 0$, then $u$ is holomorphic in both variables, so it is holomorphic in $B_{2}$. Suppose (16) is true. If we take $\partial / \partial \bar{z}_{1}$ on both sides of (16) then we have $R u=0$ by (14). Therefore $u$ is antiholomorphic in $B_{2}$ by a theorem of Forelli [3]. This completes the proof.

\section{M-HARMONIC FUNCTIONS WITH PLURIHARMONIC SQUARE}

Finally we prove that any $\mathcal{M}$-harmonic function with pluriharmonic square is either holomorphic or antiholomorphic.

Theorem 3. Suppose $\widetilde{\Delta} u=\widetilde{\Delta} u^{2}=0$ in $B_{n}$. If $\Delta u^{2}=0$, in addition, then one of $u$ and $\bar{u}$ is holomorphic in $B_{n}$. In other words, if $\mathcal{M}$-harmonic function $u$ has a pluriharmonic square, then either $u$ or $\bar{u}$ is holomorphic.

Proof: Since $u^{2}$ is pluriharmonic, it can be written as $u^{2}=f+\bar{g}$, where $f$ and $g$ are holomorphic in $B_{n}$. Hence $u=(f+\bar{g})^{1 / 2}$, a branch, where $u$ does not vanish. Since

$$
\frac{\partial^{2} u}{\partial z_{j} \partial \bar{z}_{k}}=-\frac{1}{4}(f+\bar{g})^{-3 / 2} \frac{\partial f}{\partial z_{j}} \frac{\overline{\partial g}}{\partial z_{k}}
$$

we have

$$
\begin{aligned}
0 & =\sum_{j, k=1}^{n}\left(\delta_{j k}-z_{j} \bar{z}_{k}\right) \frac{\partial^{2} u}{\partial z_{j} \bar{\partial} z_{k}} \\
& =-\frac{1}{4}(f+\bar{g})^{-3 / 2} \sum_{j, k=1}^{n}\left(\delta_{j k}-z_{j} \bar{z}_{k}\right) \frac{\partial f}{\partial z_{j}} \frac{\overline{\partial g}}{\partial z_{k}} .
\end{aligned}
$$

Therefore

$$
\sum_{j, k=1}^{n}\left(\delta_{j k}-z_{j} \bar{z}_{k}\right) \frac{\partial f}{\partial z_{j}} \frac{\bar{\partial} g}{\partial z_{k}}=0
$$


where $u$ does not vanish and so $u$ does not vanish everywhere by continuity. We apply the result of Ahern and Rudin [1, Theorem I]. If $n=2$, then one of $f$ and $g$ is a constant function, and so one of $u$ and $\bar{u}$ is holomorphic. Assume $n \geqslant 3$, and suppose that neither $f$ nor $g$ is a constant function. Then there exist

(i) an interger $m, 2 \leqslant m \leqslant n-1$,

(ii) a utitary transformation $U: \mathbb{C}^{n} \rightarrow \mathbb{C}^{n}$,

(iii) entire functions $\phi: \mathbb{C}^{m-1} \rightarrow \mathbb{C}$ and $\psi: \mathbb{C}^{n-m} \rightarrow \mathbb{C}$, such that

$$
f(U z)=\phi\left(\frac{z_{2}}{1-z_{1}}, \cdots, \frac{z_{m}}{1-z_{1}}\right), g(U z)=\psi\left(\frac{z_{m+1}}{1-z_{1}}, \cdots, \frac{z_{n}}{1-z_{1}}\right) .
$$

Therefore we may assume

$$
u^{2}(z)=\phi\left(\frac{z_{2}}{1-z_{1}}, \cdots, \frac{z_{m}}{1-z_{1}}\right)+\bar{\psi}\left(\frac{z_{m+1}}{1-z_{1}}, \cdots, \frac{z_{n}}{1-z_{1}}\right)
$$

We claim that $u^{2}$ vanishes somewhere on $B_{n}$. First we can choose $\zeta^{(1)} \in \mathbb{C}^{m-1}$ and $\zeta^{(2)} \in \mathbb{C}^{n-m}$ so that $\phi\left(\zeta^{(1)}\right)+\bar{\psi}\left(\zeta^{(2)}\right)=0$. We take a value $x_{1}$ with $0<x_{1}<1$ so that $\left|\zeta^{(1)}\right|+\left|\zeta^{(2)}\right|<R=\sqrt{1-x_{1}^{2}} / \sqrt{2}\left(1-x_{1}\right)$ and set

$$
\begin{aligned}
& z^{(1)}=\frac{\sqrt{1-x_{1}^{2}}}{\sqrt{2}} \xi^{(1)}, \xi^{(1)} \in \mathbb{C}^{m-1},\left|\xi^{(1)}\right|<1, \\
& z^{(2)}=\frac{\sqrt{1-x_{1}^{2}}}{\sqrt{2}} \xi^{(2)}, \xi^{(2)} \in \mathbb{C}^{n-m},\left|\xi^{(2)}\right|<1 .
\end{aligned}
$$

Then $\left(x_{1}, z^{(1)}, z^{(2)}\right) \in B_{n}$. We can choose $\xi^{(1)}$ and $\xi^{(2)}$ so that

$$
\begin{aligned}
\frac{z^{(1)}}{1-x_{1}} & =\frac{\sqrt{1-x_{1}^{2}}}{\sqrt{2}\left(1-x_{1}\right)} \xi^{(1)}=\zeta^{(1)} \\
\frac{z^{(2)}}{1-x_{1}} & =\frac{\sqrt{1-x_{1}^{2}}}{\sqrt{2}\left(1-x_{1}\right)} \xi^{(2)}=\zeta^{(2)} .
\end{aligned}
$$

Therefore

$$
u^{2}\left(x_{1}, z^{(1)}, z^{(2)}\right)=\phi\left(\zeta^{(1)}\right)+\bar{\psi}\left(\zeta^{(2)}\right)=\dot{0}
$$

For fixed $x_{1}$ and $z^{(2)}, u^{2}\left(x_{1}, z^{(1)}, z^{(2)}\right)$ is holomorphic in $z^{(1)}$ and hence $u^{2}$ takes all values of a neighbourhood of 0 . Therefore it cannot have a continuous square root function $u$, which is a contradicion. This shows that either $f$ or $g$ must be a constant function. That is, one of $u$ or $\bar{u}$ is holomorphic. This completes the proof. 


\section{REFERENCES}

[1] P.R. Ahern and Walter Rudin, 'M-harmonic products', Indag. Math. 2 (1991), 141-147.

[2] G.B. Folland, 'Spherical harmonic expansion of the Poisson-Szago kernel for the ball', Proc. Amer. Math. Soc. 472 (1975), $401-408$.

[3] Walter Rudin, Function theory in the unit ball of $\mathbb{C}^{n}$ (Springer-Verlag, Berlin, Heidelberg, New York, 1980).

Department of Mathematics

Korea Advanced Institute of Science and Technology

373-1 Kusong-Dong Yusong-Gu

Taejon 3-5-701

Kores 